\title{
Multi-scale and Hierarchical Description using Energy Controlled Active Balloon Model
}

\author{
Hiroshi Matsuo, Masataka Kimura and Akira Iwata \\ Nagoya Institute of Technology \\ Dept. of Electrical and Computer Eng. \\ Gokiso, Showa, Nagoya 466-8555, JAPAN \\ matsuo@elcom.nitech.ac.jp
}

\begin{abstract}
A novel multi-scale tree construction algorithm for three dimensional shape using "Energy Controlled Active Balloon Model(EC-ABM)" is proposed. The key idea is that (1) internal and external energies and a number of surfaces which make up the $A B M$ are controlled at each shrinking steps, and (2) each converged shape is regarded as one multi-scale shape. Some experimental three dimensional recognition result of the proposed method with human face data are shown.
\end{abstract}

\section{Introduction}

When humans recognize the shape of the object, rough observation of whole object shape and detail observation of partial shape are used at the same time. If these procedures are applied to recognition algorithm for computer vision, flexible and robust matching can be achieved. Many recognition algorithm for one dimensional signal and shapes contours using multi-scale data were proposed[1]. Shape of 3D object are transformed into multi-scale representation in order to observe each discrete portion of the shape contours at different view scales. The reason for using multi-scale representation is that the inflection point, which is an important characteristic information for image recognition, is increased monotonically by increasing the resolution.

While making 3-D multi scale resolution images, convolution with Gaussian function is commonly used. However features such as an inflection point does not increase monotonically by increasing the resolution. Consequently multi-scale tree structure which has suitable feature for matching is impossible to construct.

In recent years, segmentation technique which com- bine a local edge extraction operation with the use of active contour models, or snakes, to perform a global region extraction have achieved considerable success for certain applications[2]. These models simulate elastic material which can dynamically conform to object shapes in response to internal forces, external forces, and user specified constraints. The result is an elegant method of linking sparse or noisy local edge information into a coherent object description. Cohen[3] proposed three dimensional extension of snake and Tsuchiya [4] also proposed three dimensional extension model "Active Balloon Model(ABM)".

In this paper, we propose a novel multi-scale tree construction algorithm for three dimensional shape using "Energy Controlled Active Balloon Model(ECABM)". The key idea is that (1) internal and external energies and a number of surfaces which make up the ABM are controlled at each shrinking steps, and (2) each converged shape is regarded as one multi-scale shape.

\section{The Active Balloon Model}

The active balloon model is a discrete dynamic deformable model constructed from a set of mobile nodes and adjustable strings which describes 3D triangle patch models from scattered 3D points in space. Each node of the ABM moves according to it's local energy iteratively and constructs 3D shape.

The ABM is a model which expands snakes into three dimensional shell structure. The initial shape of ABM has 1280 triangle patches, and it's structure has the well known geodesic dome.

The energy function which acts on each node is defined by equation(1), and the following position of each node is decided using greedy algorithm which uses only the energies of connected nodes[5]. The energy of a 
point $x$ is defined by a summation of an internal energy $E_{i n t}$ and external energy $E_{\text {ext }}$.

$$
E_{\text {point }}(x)=E_{\text {int }}(x)+E_{\text {ext }}(x)
$$

The internal energy $E_{\text {int }}$ corresponds to a smooth factor in the regularization theory which is is defined by the following equation.

$$
E_{i n t}(x)=\alpha \sum_{i=0}^{5}\left\{\max _{j \in\{\mathbf{J}\}}(\mathbf{p}(j)-\mathbf{p}(x)) e_{i}\right\}^{2}
$$

where $\mathbf{p}(x)$ denotes position vector of each node, $e_{0}, \cdots, e_{5}$ denotes unit vector of $X,-X, Y,-Y, Z$ and $-Z$ axis respectively. $\alpha$ denotes the parameters which defines the ratio of internal and external energies. J denotes sets of nodes connected to $x$. This factor controls the smoothness of connections between nodes. The external energy $E_{e x t}$ corresponds to a penalty factor. This external energy makes the node approach the object shape. During iteration procedure, the location having the smallest energy value is chosen as the new position.

The external energy is the force produced by $3 \mathrm{D}$ points in space. It is the space potential energy which is defined using Gauss function. Consequently correspondence procedure between each node and measured $3 \mathrm{D}$ points in space is eliminated.

$$
E_{e x t}(x)=\sum_{i \in\{\text { Sampling } 3 \mathrm{D} \text { points }\}}-G_{\sigma}(\mathbf{p}(i)-\mathbf{p}(x)),
$$

$G_{\sigma}(\mathbf{x})$ is defined by equation(4).

$$
G_{\sigma}(\mathbf{d})=\frac{1}{\sqrt{2 \pi} \sigma} \exp \left(-\frac{\mathbf{p}(\mathbf{d}) \cdot \mathbf{p}(\mathbf{d})}{2 \sigma^{2}}\right)
$$

where $\sigma$ is a standard deviation of the potential field.

\section{Energy Controlled Active Balloon Model}

\subsection{The Problem of Constructing Multi- scale Tree}

MEGI (More Extended Gaussian Image) [6] is a description model to represent arbitrary shape. However many MEGI elements are necessary to represent uneven or curved surfaces with accuracy, hence it is difficult to use them for recognition. As a solution, Matsuo[7] proposed a multi-scale matching model using multi-scale description by tracing the tree from the root, which corresponds to the coarsest representation, to the leaf and using a matching algorithm.

Matsuo[7] also proposed simple method to construct multi-scale tree for 3D object. Making the multi-scale tree with AS-MEGI is a "bottom up method" that is the tree is generated from high resolution to low resolution. Therefore, at low resolution, the generated multi-scale tree changed drastically when the shape of pose of object is slightly changed. Consequently, the feature of multi-scale tree, (i.e. (1) detail shape of the object is shown in a high resolution, (2) the outline shape which neglect the object deformation and object displacement are shown in low resolution) are lost. Figure 1 shows normal vector description for a circle in low resolution. If the phase of polygon is moved a little bit, features of the set of the normal vectors changes drastically. However if making a multi-scale tree using a "top down method", these problems can be solved.

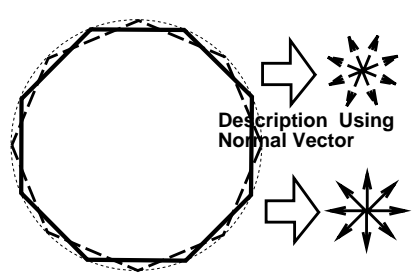

Figure 1. The normal vector description for a circle in low resolution

\subsection{Multi-scale Description using EC-ABM}

The key idea of this paper is that each shrinking step of the ABM is regarded as a multi-scale description. The the initial shape of EC-ABM changed from 1280 triangle patches to icosahedron. By making iteration procedure, initial icosahedron shape converges to a certain shape. This converged shape is regarded as one scale of a multi-scale description. To increase the resolution, each triangle element is divided into four subdividing triangle elements and iteration procedure is continued. However, a lot of calculation is needed for recognition if unconditional division is used. A division method will be described in 4 . This subdividing operation enable the ABM to describe a more detail shape description. Parameters which controls shrinking process of ABM are changed for constructing a robust multi-scale description against noise and partial shape difference. 
Three parameters, the ratio of internal and external parameters $\alpha$ in equation (2), the variance of potential distribution of external energy $\sigma$ in equation (4), and the number of triangle patch that makes up the active balloon model which controls the multi-scale resolution, are defined.

\subsection{External Energy Control}

In order to get smooth shrinking procedure, spatial potential defined by equation (4) is controlled. When constructing low resolution shape, standard deviation $\sigma$ is set to be small (this means space potential is decreased gently). To increase the resolutions, $\sigma$ is increased. Therefore, movement of each node depends on the general shape at low resolution and also depends on the partial shape at high resolution. When the parameter $\sigma$ sets large, the external potential represents a blurred object shape. On the other hand, if it is small, the distribution of potential is similar to the object shape.

\subsection{Internal Energy Control}

Changing the ratio $\alpha$ in equation (2) between the external energy and the internal energy, smoothness (discontinuity) factor can be controlled. Therefore, during low resolution constructing procedure (the first stage of shrinking), the external energy is dominated by the internal energy. With the progress of the shrinking, the ratio of internal energy becomes high. As a result, exact $3 \mathrm{D}$ shape can be reconstructed.

\section{Constructing Multi-scale Tree}

In Sec 2, the algorithm to generate a 3D multi-scale space using EC-ABM was discussed. The 3D multiscale tree can not be constructed because there are no relation between each triangle patches of each resolutions. In this section, the method of composing 3D multi-scale tree from a low resolution to a high resolution is proposed.

When the resolution is increased by dividing single triangle into four, the hierarchical relations between these triangles are defined. Doing this operation from low resolution to high resolution, multi-scale tree can be constructed.

However when all triangles are divided into four, triangles which describes flat part of the object are almost motionless after dividing. These division cases not only becomes tedious expression, but also matching candidate are increased for recognition. Useless division is limited by the following division rule of triangle patch.
1. Let three vertices of a triangle patch $\mathbf{T}$ be $P_{a}, P_{b}, P_{c}$ respectively.

2. New vertex $P_{a b}$ is added to the middle of $P_{a}$ and $P_{b} \quad P_{b c}$ and $P_{c a}$ are added similarly.

3. Calculate summation of these external energies $e_{1}$.

$$
e_{1}=E_{e x t}\left(P_{a b}\right)+E_{e x t}\left(P_{b c}\right)+E_{e x t}\left(P_{c a}\right)
$$

4. The vertex $P_{a b}$ is moved in the direction where external energy becomes low. Let new point be $P_{a b}^{\prime} . P_{b c}^{\prime}, P_{c a}^{\prime}$ are also calculated.

5. Summation $e_{2}$ of these three node is calculated.

$$
e_{2}=E_{e x t}\left(P_{a b}^{\prime}\right)+E_{e x t}\left(P_{b c}^{\prime}\right)+E_{e x t}\left(P_{c a}^{\prime}\right)
$$

6. If $e_{1}-e_{2}<T h$, then this division procedure from patch $\mathbf{T}$ to patches $\mathbf{T}_{\mathbf{1}}, \mathbf{T}_{\mathbf{2}}, \mathbf{T}_{\mathbf{3}}$ and $\mathbf{T}_{\mathbf{4}}$ is stopped else this division step is confirmed. where $T h$ is threshold parameter.

7. The above mentioned steps are performed to all triangle patches.

Figure 2 shows a division step. This figure shows that flat areas are not divided into high resolution triangles. The outline of the algorithm to construct multi-

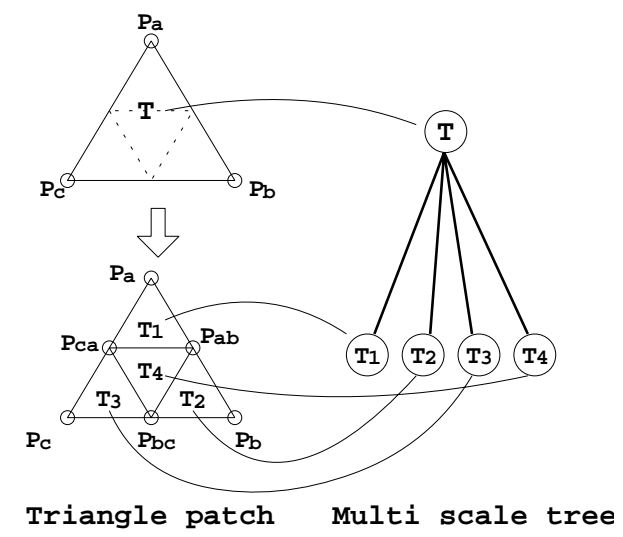

Figure 2. The division step and constructing multi-scale tree

scale shape and multi-scale tree using EC-ABM are shown as follows.

Step1 The initial shape of active balloon model is set in icosahedron. Let the initial value of these parameter $\sigma, \alpha$ be $\sigma_{0}, \alpha_{0}$ respectively. 
Step2 Do iteration procedure until a shape converges to a certain shape.

Step3 This converged shape is regarded as one scale of multi-scale description.

Step4 If present resolution attains to maximum resolution, then exit this procedure.

Step5 All triangle patches are divided into four new triangle patches, and check the division rule as described in 4 .

Step6 New $\alpha$ is set to $\alpha / \Delta \alpha$, new $\sigma$ is set to $\sigma-\Delta \sigma$, and goto Step2.

\section{The Experiment}

The experiment was performed with the range data of human full face data ( 25 faces) produced by the National Research Council Canada(NRCC)[8]. Hair part of each full face data was eliminated by hand. In this experiment, 3-D shape model(CAD model) was also generated using range data. Changing view points of the range data were also rebuilt using the original range data. Figure 3 shows one human full face data. Figure 4 shows multi-scale shapes of each resolutions shown in Figure 3 .

In this matching experiment, a view angle of elevation is fixed at 0 degrees, an azimuthal angle is changed from 0 degree to 10 degrees respectively, and range data was measured. In all experiments, parameters $\alpha_{0}, \sigma-0$ ware set at 3.5 and 10 ,respectively. $\Delta \alpha$ and $\Delta \sigma$ are set at 2 and 0.5 respectively, and five scales ware constructed.

Figure 5 shows correlation coefficient between one face in Figure 3 with no rotation and all 25 faces when the rotation angle was changed. Upper figure shows the correlation of coefficient using bottom up method, lower figure shows using proposed top down method using AS-MEGI. The correlation coefficients between correct pair are plotted, and the correlation coefficients to other 24 faces are plotted only the maximum, the minimum, the average and the standard deviation values. If max value exceed to the correlation coefficients between the correct pair, recognition is considered to fail at this rotation angle. The correlation coefficient was calculated by the multi-scale tree matching algorithm proposed by Matsuo[7]. When rotation angle is increased, recognition rate become low. This is because original face range data is not a complete 3-D image. Therefore rotation image has a lot of occluded part compared with the original image. The proposed top down method obtained a higher correlation coefficients

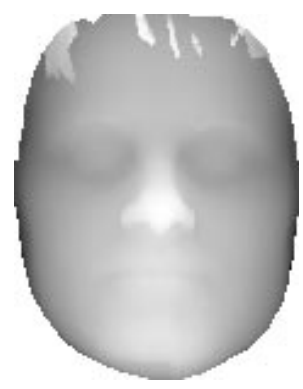

Figure 3. An example of range data images used in experiments.

than bottom up method at all rotation angle and for correct and incorrect matching pair. The reason is that all low resolution shape which was constructed by top down method becomes almost the same shape. However using the bottom up method, all low resolution shapes become quite different shape.

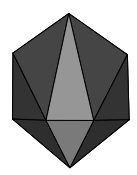

Scale.0

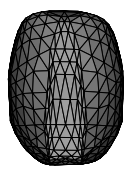

Scale.3

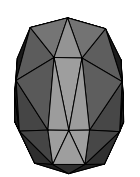

Scale.1

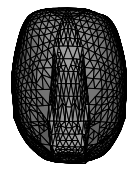

Scale.4
Figure 4. multi-scale face

Figure 6 shows recognition rate using the top down multi-scale tree construction algorithm using ASMEGI and bottom up algorithm which was used in [7]. Recognition result is defined as the maximum correlation coefficient between the rotated angle data and the original data. Using top down method, recognition rate becomes $100 \%$ for all rotation angle. These results shows extremely high recognition ability of top down method, even if applying to the images contains curved surface which have very few features like human faces. 


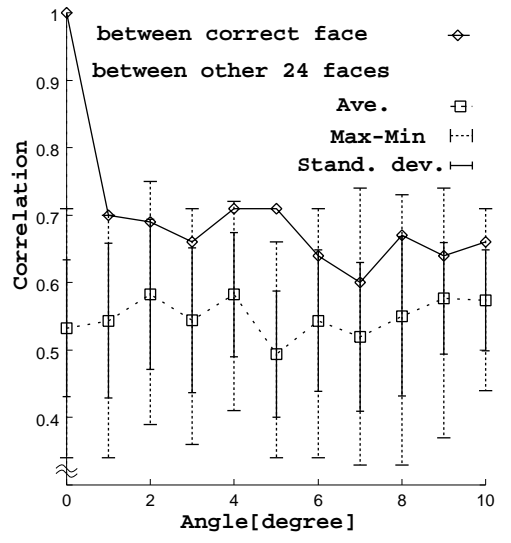

(a) Using bottom up method

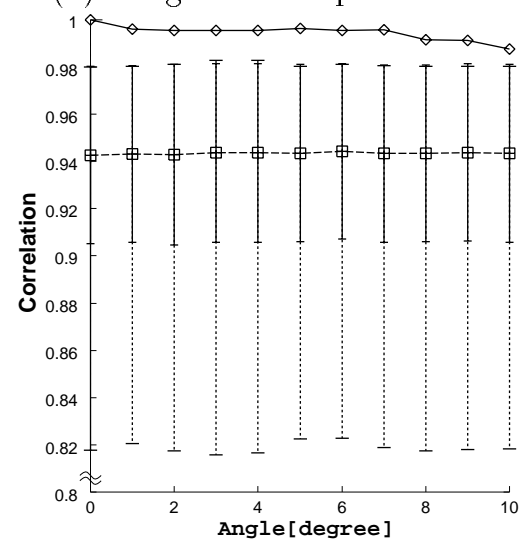

(b) Using proposed bottom up method

Figure 5. The relationship between direction angle and correlation coefficient

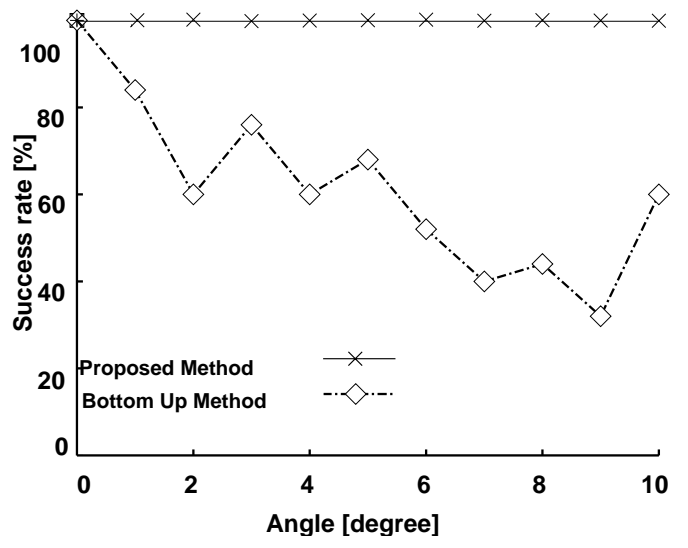

Figure 6. The relationship between direction angle and success rate

\section{Conclusion}

We proposed an algorithm for generating multi scale resolution with tree data structure using energy controlled active balloon model. This algorithm uses shrinking procedure of active balloon model and controlling the number of nodes, the external energy and the internal energy. We also experimented with the proposed algorithm and fairly good result had been achieved.

\section{References}

[1] M.Leyton.A process-grammar for shape. Artificial Intell., 34:213-247,1988.

[2] M.Kass, A.Witkin and D.Terzopoulos. Snakes: Active Contour Models. Int. J. Comput. Vision, 1(4):321-331, 1988.

[3] L.D. Cohoen. On active contour models and balloons. In CVGIP: Image Understanding, 53(2):211218, 1991.

[4] K.Tsuchiya, H.Matsuo, A.Iwata. 3D Shape Reconstruction from Range Data Using Active Balloon Model and Symmetry Restriction. Trans. of Institute of Elec. Info. and Comm. Eng. (in J), J76-DII(9):1967-1976, Sep. 1993.

[5] Williams D.J. and Shah M. A Fast Algorithm for Active Contours. In Proc. of Third Int. Conf. on Comput. Vision, 592-595, 1990.

[6] H.Matsuo and A.Iwata. 3-D Object Recognition using MEGI Model From Range Data. 12th Int. Conf. on Pattern Recognition(ICPR), I:843-846, Sep. 1994.

[7] H.Matsuo, J.Funabashi and A.Iwata. 3D Object Recognition using Adaptive Scale MEGI. 13th Int. Conf. on Pattern Recognition(ICPR), IV:117-122. Aug. 1996.

[8] Rioux M., and Cournoyer L. The NRCC Threedimensional Image Data Files. The Report CNRC 29077, National Research Council Canada, Ottwa, Canada, 1988. 